\title{
UNITARY PARTS OF CONTRACTIVE HANKEL MATRICES
}

\author{
JEFFREY R. BUTZ
}

\begin{abstract}
For a Hankel matrix $H=\left(c_{j+k}\right)$ which is a contraction, necessary and sufficient conditions are obtained for the existence of a nontrivial unitary part, and an explicit description of this unitary part is given.
\end{abstract}

1. Introduction. In what follows, $l^{2}$ will denote the usual class of squaresummable complex sequences, $L^{2}$ will denote the class of Lebesgue measurable functions on the unit circle in the complex plane, and $H^{2}$ will denote the Hardy (closed) subspace of $L^{2}$ consisting of those functions in $L^{2}$ whose Fourier coefficients vanish on the negative integers. Functions which differ only on zero sets will be considered equal.

With a sequence $\left(c_{0}, c_{1}, \ldots\right)$ in $l^{2}$ we associate the Hankel matrix $H=$ $\left(c_{j+k}\right)$, where $j, k=0,1,2, \ldots$, acting on $l^{2}$. If we define $\phi\left(e^{i \theta}\right)=c_{0}+$ $c_{1} e^{i \theta}+c_{2} e^{2 i \theta}+\ldots$, then such a matrix may be realized as an operator on $H^{2}$, defined by

$$
H x\left(e^{i \theta}\right)=P_{+} \phi\left(e^{i \theta}\right) x\left(e^{-i \theta}\right),
$$

where $P_{+}: L^{2} \rightarrow H^{2}$ is the orthogonal projection.

A result of Nehari [4] states that a Hankel matrix $H$ is bounded if and only if there exists a function $f \in L^{\infty}$ such that

$$
c_{n}=\frac{1}{2 \pi} \int_{0}^{2 \pi} f\left(e^{i \theta}\right) e^{-i n \theta} d \theta,
$$

and, in this case, $f$ can be chosen so that $\|f\|_{\infty}=\|H\|$. Such a function $f$ is called a minifunction of $H$.

We shall consider Hankel matrices which are contractions $(\|H x\| \leqslant\|x\|)$ on the Hardy space $H^{2}$. If a Hankel matrix is a contraction, then, by the theorem of Nehari, we can find a minifunction $f \in L^{\infty}$ such that $\|f\|_{\infty}=$ $\|H\| \leqslant 1$ and $H x\left(e^{i \theta}\right)=P_{+} f\left(e^{i \theta}\right) x\left(e^{-i \theta}\right)$ for $x \in H^{2}$.

2. The unitary part of a Hankel contraction. Following Sz.-Nagy and Foiaş [5], we say that a contraction $T$ on a Hilbert space $K$ is completely nonunitary if $T$ has no nontrivial reducing subspace $N$ such that the restriction $T \mid N$ of $T$ to $N$ is unitary. It is known [5, Theorem I.3.2] that for any contraction $T$ on $K$ we can find a unique orthogonal decomposition $K=M \oplus M_{1}$ such that $T \mid M$ is unitary and $T \mid M_{1}$ is completely nonunitary. It is not excluded that $M$ or

Received by the editors January 31, 1977.

AMS (MOS) subject classifications (1970). Primary 47A20, 47B35.

(1) American Mathematical Society 1977 
$M_{1}$ is possibly the subspace $\{0\}$. Furthermore, $M$ is given by $M=\{x \in K$ : $\left.\left\|T^{n} x\right\|=\|x\|=\left\|T^{* n} x\right\|, n=1,2 \ldots\right\}$ and is called the unitary subspace of $T . T \mid M$ is called the unitary part of $T$.

If $\psi\left(e^{i \theta}\right) \in L^{\infty}$, then the corresponding bounded Toeplitz operator $T_{\psi}$ : $H^{2} \rightarrow H^{2}$ is defined by $T_{\psi}: x\left(e^{i \theta}\right) \mapsto P_{+} \psi\left(e^{i \theta}\right) x\left(e^{i \theta}\right)$ for $x \in H^{2}$. In [1] Goor proved that if $T_{\psi}$ is a Toeplitz contraction $\left(\left\|T_{\psi}\right\| \leqslant 1\right.$, i.e., $\left|\psi\left(e^{i \theta}\right)\right| \leqslant 1$ a.e. $)$, then $T_{\psi}$ is completely nonunitary unless $\psi$ is a constant. This result may be used to obtain necessary and sufficient conditions for the existence of a nontrivial unitary part of a Hankel contraction. These conditions, together with a characterization of the unitary part, when it exists, are obtained as a corollary to the following.

THEOREM 2.1. Let $H=\left(c_{j+k}\right)$ be a Hankel contraction. Then $H$ will have a nontrivial unitary subspace $M$ only when there exists a minifunction $f\left(e^{i \theta}\right)$ for $H$ such that

$$
\begin{gathered}
\left.\left|f\left(e^{i \theta}\right)\right|=1 \text { a.e. (so that }\|H\|=1\right) \text {, and } \\
f\left(e^{i \theta}\right) f\left(e^{-i \theta}\right)=k^{2} \text { a.e. for some constant } k^{2},|k|=1 .
\end{gathered}
$$

In such a case, the minifunction $f\left(e^{i \theta}\right)$ is unique, and $M$ is given by the three equivalent expressions:

$$
M=\left[e^{i \theta} f\left(e^{i \theta}\right) H^{2}\right]^{\perp} \cap H^{2},
$$

where the orthogonal complement is in $L^{2}$,

$$
\begin{aligned}
& M=\left\{x \in H^{2}: H^{*} H x=x\right\}, \\
& M=\left\{x \in H^{2}: \bar{k} H x=x\right\} \oplus\left\{x \in H^{2}: \bar{k} H x=-x\right\} .
\end{aligned}
$$

Proof. Suppose that a Hankel contraction $H$ has a nontrivial unitary subspace. Then there exists some $x \neq 0$ in $H^{2}$ such that for $H$ and its adjoint $H^{*}=\bar{H}$ we have $\left\|H^{n} x\right\|=\|x\|=\left\|\bar{H}^{n} x\right\|$ for $n=1,2, \ldots$ Taking $n=1$ gives that

$$
\|x\|=\|H x\|=\left\|P_{+} f\left(e^{i \theta}\right) x\left(e^{-i \theta}\right)\right\| \leqslant\left\|f\left(e^{i \theta}\right) x\left(e^{-i \theta}\right)\right\| \leqslant\left\|x\left(e^{-i \theta}\right)\right\| .
$$

This implies that $P_{+} f\left(e^{i \theta}\right) x\left(e^{-i \theta}\right)=f\left(e^{i \theta}\right) x\left(e^{-i \theta}\right)$, so that $H x=$ $f\left(e^{i \theta}\right) x\left(e^{-i \theta}\right) \in H^{2}$. It also immediately follows that the minifunction $f\left(e^{i \theta}\right)$ must therefore be unique, cf. [2, p. 863]. Furthermore, we may apply a well-known corollary of the F. and M. Riesz theorem [3, p. 52] to the equality

$$
\left\|f\left(e^{i \theta}\right) x\left(e^{-i \theta}\right)\right\|=\left\|x\left(e^{-i \theta}\right)\right\|
$$

to conclude that $\left|f\left(e^{i \theta}\right)\right|=1$ almost everywhere on the unit circle. This establishes (2.1).

Taking now $n=2$, we get $\|x\|=\left\|H^{2} x\right\|=\left\|H f\left(e^{i \theta}\right) x\left(e^{-i \theta}\right)\right\|=\| P_{+}$ $f\left(e^{i \theta}\right) f\left(e^{-i \theta}\right) x\left(e^{i \theta}\right)\|\leqslant\| f\left(e^{i \theta}\right) f\left(e^{-i \theta}\right) x\left(e^{i \theta}\right)\|\leqslant\| x\left(e^{i \theta}\right) \|$, so that

$$
H^{2} x=f\left(e^{i \theta}\right) f\left(e^{-i \theta}\right) x\left(e^{i \theta}\right)=T_{\psi} x,
$$

where $\psi\left(e^{i \theta}\right)=f\left(e^{i \theta}\right) f\left(e^{-i \theta}\right)$. Continuing, we obtain 


$$
\begin{aligned}
H^{2 n+1} x\left(e^{i \theta}\right) & =f^{n+1}\left(e^{i \theta}\right) f^{n}\left(e^{-i \theta}\right) x\left(e^{-i \theta}\right), \quad n=0,1, \ldots, \\
H^{2 n} x\left(e^{i \theta}\right) & =\left[f\left(e^{i \theta}\right) f\left(e^{-i \theta}\right)\right]^{n} x\left(e^{i \theta}\right), \quad n=1,2, \ldots
\end{aligned}
$$

But (2.7) together with its analogue for $\bar{H}^{2 n}$ (obtained by replacing $f\left(e^{i \theta}\right)$ by $\left.\bar{f}\left(e^{i \theta}\right)\right)$ implies that the Toeplitz operator $T_{\psi}$ has a nontrivial unitary part. Therefore, by Goor's result, $f\left(e^{i \theta}\right) f\left(e^{-i \theta}\right)=k^{2}$ for some constant $k,|k|=1$. Hence, $\bar{k} f\left(e^{i \theta}\right)=k \bar{f}\left(e^{-i \theta}\right)$, so that, by $(1.1), \bar{k} c_{n}$ is real for all $n$.

Formulas (2.6) and (2.7) now reduce to

$$
\begin{aligned}
H^{2 n+1} x\left(e^{i \theta}\right) & =k^{2 n} H x\left(e^{i \theta}\right), \quad n=0,1,2, \ldots, \\
H^{2 n} x\left(e^{i \theta}\right) & =k^{2 n} x\left(e^{i \theta}\right), \quad n=1,2, \ldots,
\end{aligned}
$$

valid for all $x$ in the unitary subspace of $H$. Similar expressions are easily obtained for $\bar{H}^{2 n+1} x\left(e^{i \theta}\right)$ and $\bar{H}^{2 n} x\left(e^{i \theta}\right)$.

The maximal subspace on which $H$ is unitary now becomes $M=\{x \in$ $\left.H^{2}:\|H x\|=\|x\|\right\}=\left\{x \in H^{2}: H^{*} H x=x\right\}$, giving (2.4). Furthermore, we then have $x \in M \Leftrightarrow f\left(e^{i \theta}\right) x\left(e^{-i \theta}\right) \in H^{2} \Leftrightarrow f\left(e^{-i \theta}\right) x\left(e^{i \theta}\right) \perp e^{i \theta} H^{2} \Leftrightarrow$ $x\left(e^{i \theta}\right) \perp e^{i \theta \bar{f}}\left(e^{-i \theta}\right) H^{2} \Leftrightarrow x \in\left[e^{i \theta} f\left(e^{i \theta}\right) H^{2}\right]^{\perp} \cap H^{2}$, since $f\left(e^{-i \theta}\right)=k^{2} \bar{f}\left(e^{i \theta}\right)$. This establishes (2.3). Finally, since the matrix $\bar{k} H$ is real, hence selfadjoint, with $\|\bar{k} H\|=1$, we get (2.5). This completes the proof.

As a result of the above theorem, we then obtain a characterization of those Hankel matrices having nontrivial unitary subspaces.

Corollary 2.1. Let $H$ be a Hankel contraction. Then a necessary and sufficient condition that $H$ have a nontrivial unitary subspace is that there exist a constant $k,|k|=1$, such that $\bar{k} H$ is real (hence selfadjoint) and that $M$ in (2.5) satisfy $M \neq\{0\}$.

Using a result of Sz.-Nagy and Foiaş for completely nonunitary contractions, we can define a functional calculus for functions $u \in H^{\infty}$. In particular, if $B\left(H^{2}\right)$ is the space of bounded operators on $H^{2}$, we then have the following.

COROLlary 2.2. Let $H$ be a Hankel contraction. If either $\|H\|<1$ of $k H$ is not real for all $k \in \mathrm{C}$, then the map $u \rightarrow u(H)$ from the Hardy space $H^{\infty}$ into $B\left(H^{2}\right)$ defined by

$$
u(H)=\underset{r \rightarrow 1-}{\text { strong } \lim } \sum_{k=0}^{\infty} a_{k} r^{k} H^{k},
$$

where $u\left(e^{i \theta}\right)=\sum_{k=0}^{\infty} a_{k} e^{i k \theta}$, is a contractive homomorphism of the algebra $H^{\infty}$ into $B\left(H^{2}\right)$.

This follows from the above theorem and Theorem III.2.1 of [5].

Theorem 2.1 can also be used to establish a property of the point spectrum of a bounded Hankel matrix.

Corollary 2.3. Let $H$ be a bounded Hankel matrix. If $\lambda=\|H\|$ is an eigenvalue of $H$, then $H$ is real. Hence, if $\alpha H$ is not real for any nonzero 
(complex constant) $\alpha$, then $|\lambda|<\|H\|$ holds for all eigenvalues $\lambda$ of $H$ (if any).

Proof. Without loss of generality, we can suppose that $\|H\|=1$. (In such a case, the associated eigenspace $M=\left\{y \in H^{2}: H y=y\right\}$ is well-known to be a reducing subspace for the operator $H$ [5, Proposition I.3.1].) If $y \neq 0$ satisfies $H y=y$, then, by the proof of Theorem $2.1, \bar{H}=\bar{\alpha} H$ and $H^{2} y=\alpha y$ for some $|\alpha|=1$. But since $H^{2} y=y$, we get $\alpha=1$, and hence $\bar{H}=\bar{\alpha} H=H$, which shows that $H$ must be real.

ACKnowledgement. The author wishes to thank Professor Philip Hartman for his encouragement and many helpful suggestions.

\section{REFERENCES}

1. R. Goor, On Toeplitz operators which are contractions, Proc. Amer. Math. Soc. 34 (1972), 191-192.

2. P. Hartman, On completely continuous Hankel matrices, Proc. Amer. Math. Soc. 9 (1958), 862-866.

3. K. Hoffman, Banach spaces of analytic functions, Prentice-Hall, Englewood Cliffs, N. J., 1962.

4. Z. Nehari, On bounded bilinear forms, Ann. of Math. (2) 65 (1957), 153-162.

5. B. Sz.-Nagy and C. Foias, Harmonic analysis of operators on Hilbert space, North-Holland, Amsterdam, 1970.

Department of Mathematics, The Johns Hopkins University, Baltimore, Maryland 21218

Department of Mathematics, The University of OkLahoma, Norman, Okiahoma 73061 (Current address) 